A N N A L E S Annales de Bretagne et des Pays de l'Ouest

Anjou. Maine. Poitou-Charente. Touraine

108-3 | 2001

Varia

\title{
Le saumon, un poisson convoité
}

Braconnages et braconniers en Bretagne sous l'Ancien Régime

\section{Pierre Martin}

\section{(2) OpenEdition}

\section{Journals}

Édition électronique

URL : http://journals.openedition.org/abpo/1695

DOI : $10.4000 /$ abpo.1695

ISBN : 978-2-7535-1483-6

ISSN : 2108-6443

\section{Éditeur}

Presses universitaires de Rennes

\section{Édition imprimée}

Date de publication : 20 septembre 2001

Pagination : $57-67$

ISBN : 978-2-86847-625-8

ISSN : 0399-0826

\section{Référence électronique}

Pierre Martin, «Le saumon, un poisson convoité », Annales de Bretagne et des Pays de l'Ouest [En ligne], 108-3 | 2001, mis en ligne le 20 septembre 2003, consulté le 02 mai 2019. URL : http:// journals.openedition.org/abpo/1695; DOI : 10.4000/abpo.1695 


\title{
Le saumon, un poisson convoité Braconnages et braconniers en Bretagne sous l'Ancien Régime
}

\author{
Pierre MARTIN \\ Professeur certifié d'histoire-géographie \\ Lycée Auguste-Brizeux à Quimper \\ Doctorant, SoliTo-Université de Bretagne sud
}

Le saumon, poisson royal par définition, jouissait, sous l'Ancien Régime, d'une excellente réputation auprès des poissonniers et des consommateurs et sa pêche était très réglementée. Considéré comme le roi des poissons par Izaak Walton, dans son remarquable traité de pêche écrit en 1653 et publié en 1676, le saumon était au cœur des discordes entre seigneuries voisines et suscitait des envies pouvant conduire au braconnage $^{1}$. Sa chair, très estimée et digne des tables royales et princières, se monnayait fort cher ${ }^{2}$. Les saumons, poussés par le besoin de se reproduire, remontaient les fleuves côtiers bretons en masse pour regagner les frayères : c'est alors qu'ils se heurtaient aux pièges licites et illicites tendus par les hommes.

\section{Les rivières à saumon de Bretagne}

On ne compte pas moins de 21 rivières à saumon en Bretagne, dont 11 se jettent sur la côte nord (en y comprenant le Couesnon), et sur la côte sud. Tous ces cours d'eau ont un caractère commun : ce sont des petites rivières, mais certaines peuvent être très productives. Les bonnes performances de la Bretagne, sur le plan des remontées de saumons, lui viennent en majeure partie de caractéristiques géographiques nettement favorables par rapport au reste de la France. La région, qui bénéficie d'un climat dans lequel les températures dépassent rarement les 20 degrés, peut compter sur une forte pluviométrie, sur des rivières où

1. WALTON Izaak, The compleat angler or The contemplative man's recreation, Complete and Unabrigded, Wordsworth Editions Limited, 1996, p. 180.

2. PATte Jean-Yves et QuENEAU Jacqueline, Mémoire gourmande de Madame de Sévigné, Paris, éd. du Chêne, 1996, p. 152. 
les plats succèdent à quelques petits rapides, donc sur un grand nombre de rivières à saumon. Dans la seule Cornouaille, l'Aulne, l'Odet, le Jet, le Steïr, la Douffine, le Goyen, l'Aven, l'Ellé et l'Isole rassemblent un cheptel salmonicole important. Même si certains spécialistes ont encore beaucoup de difficultés à chiffrer approximativement les remontées de Salmo salar dans les rivières bretonnes sous l'Ancien Régime, certaines sources tendent à nous prouver son abondance et cela malgré une pollution endémique due aux activités proto-industrielles. De passage à Châteaulin au début du XVIII $^{\mathrm{e}}$ siècle, Le Masson du Parc, fraîchement nommé Commissaire aux pêches par le roi, déclare qu'on y pêche " quelquefois 20, 30 à 40 saumons en une seule marée ${ }^{3}$ ". Pour Quimperlé, ville située au confluent de l'Ellé et de l'Isole, une étude de la consommation de poisson des moines de l'abbaye de Sainte-Croix permet de mettre en évidence l'abondance et le caractère saisonnier des arrivées de saumons ${ }^{4}$. Ceuxci remontent surtout les fleuves côtiers de mai à novembre : des prix d'achat très bas soulignent l'abondance de ces arrivages. Ces variations annuelles sont aussi à mettre en relation avec le carême, temps fort des célébrations chrétiennes où le poisson royal devenait presque inabordable, ainsi qu'avec la diminution de la taille des poissons au cours de l'été, époque où les rivières regorgent de grilses ou petits saumons. Les saumons de dévalaison étaient aussi consommés, quoique moins recherchés. L'abondance de ce poisson est aussi attestée par les baux à ferme des pêcheries qui barraient les cours des fleuves côtiers. À Quimperlé, mais également sur le cours du Blavet et du Scorff, les seigneurs louaient leurs pièges contre des espèces sonnantes et trébuchantes mais également contre des saumons. Ces paiements en nature leur permettaient de se nourrir gratuitement. En 1490, le fermier des Grands Gorets, sur l'Ellé, devait à l'abbé de Sainte-Croix 20 saumons par an ${ }^{5}$. Au XVII ${ }^{\mathrm{e}}$ siècle, cette contribution tomba à quatre poissons, non par raréfaction de l'espèce, mais parce que l'abbaye préférait alors recevoir de l'argent.

L'abondance et le prix du saumon en faisaient une espèce convoitée. $\mathrm{Au} \mathrm{XVIII}{ }^{\mathrm{e}}$ siècle, il arrivait en seconde position derrière le turbot et se vendait en moyenne 3 livres. Le brochet le talonnait de près et le " grand bar " avait aussi les faveurs des palais avertis. Maquereaux, harengs et lamproies se partageaient le bas du tableau ${ }^{6}$. Toutefois son prix obéissait à de très fortes variations saisonnières. De janvier à février, de par sa raréfaction il se vendait à plus de 4 livres pièce. En mars, lors du carême, son prix s'envolait entre 5 et 6 livres. D'avril à juillet, sa valeur marchande baissait. D'avril à mi-juin remontaient des poissons de taille moyenne qui oscillaient entre 6 et 10 livres. Les " madeleinaux " ou saumons de la SaintJacques, castillons ou saumonneaux, remontaient en grand nombre de la

3. Arch. nat., Marine C5/21 f 425 .

4. Arch. dép. du Finistère, 5 H 143 à 5 H 236. La période étudiée s'étend de 1539 à 1770 .

5. Idem, $5 \mathrm{H} 40$.

6. Id., 5 H 174 à 5 H 217. 
fin du mois de juin au mois de juillet après une année passée en mer. Le niveau des eaux étant suffisant, ils remontaient aisément jusqu'aux pêcheries édifiées par les seigneurs. De la fin du mois de juin à la fin du mois de septembre, chaque pièce se négociait à un peu plus d'une livre. Les petits saumons d'été, appelés grilses ou castillons, étaient certes très nombreux mais aussi beaucoup moins gros. Leur poids oscillait entre 3 et 6 livres. Cette abondance saisonnière ne pouvait pas laisser les riverains indifférents. Avec les crues automnales remontaient les gros saumons d'hiver, moins nombreux mais plus chers ${ }^{7}$.

Les pièges, barrages, écluses et " gorets " qui barraient tous les fonds des estuaires bretons bloquaient les grands migrateurs dans leur remontée et entraînaient, fort logiquement, une concentration importante de saumons tentant de passer par-dessus ces obstacles. Ces pièges, réputés infranchissables, de par leur hauteur, accumulaient les poissons au rythme des marées. Les coffres à poisson se remplissaient et les filets étaient sans cesse relevés. Cette densité de poisson et ce débordement d'activité de la part des fermiers des pêcheries ne pouvaient que susciter jalousies et convoitises. Le saumon se vendait fort bien car sa chair était très estimée, fraîche ou salée. La vente des saumons pêchés à la pêcherie royale de Châteaulin était affermée à des négociants de la région environnante qui les revendaient ensuite à Rennes et même à Paris. Les fermiers revendeurs de cette précieuse marchandise venaient de Lamballe, Guingamp, Braspart, Châteaulin ${ }^{8}$. Ils étaient poissonniers et étaient attirés par ce marché particulièrement fructueux. En 1729, afin de vendre ses saumons dans les meilleures conditions, Mathurin Jegat, fermier des pêcheries de l'abbaye de Sainte-Croix de Quimperlé passe un contrat de vente avec un poissonnier d'Hennebont pour 180 livres $^{9}$. La revente des saumons était donc chose aisée. La facilité pour écouler ce poisson royal était un autre facteur déterminant de la fraude, tout comme tout simplement l'envie de nuire aux seigneurs et surtout à leurs représentants directs, les fermiers des pêcheries.

\section{Conflits de pêche et braconnage}

Les braconniers entraient en action lorsqu'il existait un flou juridique quant à la possession des pêcheries ou lorsque l'exercice des droits de pêche entraînaient des luttes intestines au sein des ordres dirigeants. À Quimperlé, la possession des droits de pêcheries de l'abbaye SainteCroix, dans l'Ellé, l'Isole et la Laïta suscitait les convoitises et la jalousie des moines blancs de Saint-Maurice possessionnés en aval de Quimperlé. C'est au XVIII ${ }^{\mathrm{e}}$ siècle que la querelle éclate. Lorsque Lemasson du Parc visite les bords de la Laïta en 1726, il passe chez les bernardins pour exa-

7. Id.

8. Arch. dép. du Finistère, 9 C 1/55-63-64-69-73-80-94.

9. Idem, $4 \mathrm{E} 233 / 129$. 
miner leurs titres : "Les abbés et religieux prétendent avoir un droit de pesche exclusive dans la dite rivière de Quimperlé depuis son embouchure remontant aux ponts de la dite ville ${ }^{10}$. "Ils arguent d'une bulle du pape Honorius en date du 27 août 1225. Mais il n'est pas spécifié dans quelles rivières, quels ruisseaux, quelles eaux ils peuvent faire prévaloir leurs droits. Forts de la possession de cette bulle, les domestiques de Saint-Maurice viennent donner des coups de senne et barrer la rivière avec leurs filets sous les pêcheries de l'abbaye de Sainte-Croix, le 14 octobre 1727. Les incidents s'accumulent et, le même jour, l'abbé de SaintMaurice et ses hommes viennent insulter les fermiers de Sainte-Croix ${ }^{11}$. Ces incidents sont fort courants et se renouvellent dans tous les fonds d'estuaires comme c'est le cas sur le Blavet où l'abbaye de La Joie doit lutter contre la principauté de Guémené pour finalement céder une partie de ses droits de pêche. Parfois, certains pêcheurs, envieux, tentent d'ébranler les privilèges des seigneurs riverains. En 1783, à Quimperlé, un maître de chaloupe, Louis Merrien, vient tendre ses filets près des pêcheries de l'abbaye noire. Les religieux lui demandent réparation pour les saumons qu'il a pêchés sur leurs terres, mais ce dernier exige que les moines lui présentent leurs titres ${ }^{12}$. Ce cas n'est pas isolé puisqu'il se renouvelle à Quimper, où l'évêque possède une pêcherie importante. Le 20 mars 1727, Guillaume Keraudren, pêcheur de profession, est surpris en action de pêche non loin du moulin de l'évêque ${ }^{13}$.

Ce qui vaut pour la chasse vaut également pour la pêche. En effet, si le braconnage a ses rythmes annuels, il connaît aussi de fortes variations saisonnières, voire mensuelles ${ }^{14}$. Les braconniers opèrent autant la nuit que le jour mais cela dépend encore des saisons. Lors des grandes remontées de la fin de l'hiver et du printemps, ils opèrent plutôt la nuit et n'hésitent pas à barrer la rivière avec des filets. Parfois, ils viennent chaparder du matériel de pêche. Le 3 février 1649, Colomban le Treffvou, fermier des gorêts de Quimperlé, se fait dérober devant sa porte six guideaux servant aux " petits gorets ${ }^{15}$ ". Le 31 mars 1730, Jean-Claude Pignolet, Julien Guellon et Mathurin Jegat, tous les trois fermiers des pêcheries de Quimperlé se plaignent devant le tribunal de l'Amirauté que les habitants de Lothéa, Guidel et Rédéné, bloquent la rivière avec leurs filets durant la nuit ${ }^{16}$. En avril 1729, la bande d'un certain Le Clanche agit impunément sur les bords de la Laïta au milieu de la nuit ${ }^{17}$. En mars 1727 , le fermier de l'évêque de Quimper se plaint du braconnage endémique ${ }^{18}$. En

10. Arch. nat., Marine, C 5/21, $\mathrm{f}^{\circ} 355$.

11. Arch. dép. du Finistère, 5 H 39.

12. Idem.

13. Id., 1 G 97, 20 B 4441.

14. SALVADORI Philippe, La chasse sous l'Ancien Régime, Paris, Fayard, 1996, p. 282.

15. Arch. dép. du Finistère, 19 B 227.

16. Idem, 20 B 4437.

17. Id., 20 B 4437, 20 B 4452.

18. Id., 1 G 97. 
juin 1766, le fermier des pêcheries de l'abbaye de La Joie se plaint d'un meunier qui tend des filets sous les pêcheries de l'abbesse ${ }^{19}$. Les pêcheries de saumons des seigneurs de la Roche Jagu sont aussi visitées " journellement et nocturnement " par des individus mal intentionnés. Le 5 mars 1601, un braconnier est surpris en train de tendre des filets sous la pêcherie ${ }^{20}$. Le 22 mars 1759 , Guillaume Jan, fermier et meunier des écluses de la Roche Jagu porte des menaces contre plusieurs particuliers qui s'arrogent la liberté de pêcher "nocturnement comme le jour, mesmes sur lesdites écluses et sous les pescheries ${ }^{21}$ ", si bien que le suppliant est obligé d'aller souvent de jour comme de nuit visiter ses pêcheries. Le 19 janvier 1777, Raoul Urvois, meunier et fermier de ces mêmes pêcheries se plaint des pêcheurs qui tentent de détruire ses pièges ${ }^{22}$. Au vu de ces quelques exemples, il apparaît bien que, durant le mois du carême et le début du printemps, les fermiers des pêcheries doivent être constamment sur leurs gardes. Les braconniers, peu farouches opèrent autant le jour que la nuit et poussent même le vice jusqu'à narguer les fermiers sur et sous les barrages. Même si les sources ne nous permettent pas de tirer des conclusions irréfutables, il apparaît bien que le XVIII ${ }^{\mathrm{e}}$ siècle semble être marqué par une recrudescence du braconnage sans doute lié à des mouvements contestataires embryonnaires contre l'autorité et les privilèges seigneuriaux. Il faut aussi ajouter que c'est durant cette période que la monarchie renforce sa politique de mainmise sur le littoral et tente de s'approprier ces droits en promulguant de nombreuses ordonnances ${ }^{23}$. Les rythmes de la répression démontrent qu'il s'agit d'un mal répandu et généralisé sur l'ensemble des vallées fluviales. Cependant, ce phénomène n'encombre pas les tribunaux.

Pour accomplir leurs larcins certains et disons la plupart n'hésitent pas employer les grands moyens. Ici, il ne s'agit pas de braconner du saumon au moyen d'une ligne mais c'est plutôt l'usage du filet et de ses variantes qui est généralisé. En 1727, Pierre Trousier, fermier des pêcheries de l'évêque de Quimper séquestre un filet de dix brassées qu'il a trouvé sous sa pêcherie et le " dépose en lieu sûr en attendant que le braconnier vienne le réclamer ${ }^{24}$ ". Le 23 juin 1766, Joseph Le Gallo fermier des gorets de l'abbaye de La Joie, se plaint du meunier du moulin de Porvern qui n'hésite pas à tendre des filets avec l'aide d'un bateau pour pêcher des saumons dans l'étendue prohibitive de l'abbaye. Lorsqu'il vient arracher le filet du braconnier, celui-ci est chargé de saumons ${ }^{25}$. Dans une liste des « jeunes

19. Arch. dép. du Morbihan, $60 \mathrm{H} 67$.

20. Arch. dép. des Côtes d'Armor, 108 J 42.

21. Idem.

22. Id

23. MARTIN Pierre, Les droits maritimes de l'abbaye de Sainte-Croix de Quimperlé sous l'Ancien Régime, DEA, Gérard LE BouEDEC (dir.), Université de Rennes 2/Université de Bretagne Sud, 1999, 3 vol., 518 p., dactyl.

24. Arch. dép. du Finistère, 1 G 97.

25. Arch. dép. du Morbihan, 60 H 67. 
gens avec différents enjeints peschants sur les droits de $\mathrm{M}^{\mathrm{gr}}$ le Maréchal Duc de Richelieu, pair de France " établie au XVIII ${ }^{\mathrm{e}}$ siècle, on constate que l'emploi du bateau est d'un usage courant et que les filets, "sizaux et autres carlets" ont les faveurs des fraudeurs ${ }^{26}$. D'autres n'hésitent pas à barrer totalement le cours de la rivière. En avril 1729, la bande de Le Clanche qui sévissait sur la Laïta est surprise par les fermiers au cours d'une ronde nocturne. Ils remarquent que la rivière est entièrement barrée par trois filets qui sont arrimés à des pieux.

C'est donc aux fermiers de faire la police eux-mêmes. Il faut dire qu'en face, les braconniers se laissent rarement faire. Ils sont souvent armés de bâtons et n'hésitent pas à porter des coups sur ceux qui les surprennent. Les membres de la bande de Le Clanche sont armés de bâtons ferrés et font parfois preuve d'une grande violence ${ }^{27}$. D'autres, débusqués en pleine occupation, se servent de leurs rames pour taper sur les fermiers risquant parfois de provoquer des noyades. Les fermiers sont généralement armés et font parfois feu sur leurs agresseurs. Beaucoup d'inventaires après décès mentionnent des armes à feu et quelques armes blanches comme des couteaux de chasse. Ces armes ne servaient pas qu'à décorer les linteaux des cheminées. Les altercations dépassent souvent le cadre des insultes et les fermiers, débordés et surpris par autant de violence, peuvent commettre l'irréparable. En 1729, alors qu'ils sont attaqués par une bande de braconniers qu'ils viennent de surprendre en flagrant délit, les fermiers, affolés et armés de fusils, égratignent le chef de la bande. Le rapport du chirurgien est éloquent :

" Nous avons remarqué à la partie postérieure et inférieure du fémur quatre grains de plom pénétrant dans les chairs costé gauche, plus douze grains sur la partie postérieure de la jambe à l'androit de l'articulation du fémur et du tibia ce qui nous parroit préjudicier à la suite le mouvement de cette partie ou la mettre dans le danger. Plus nous avons remarqué à la partie supérieure et postérieure du tibia du mesme costé gauche quatre grains de plom, de plus un autre grain de plom à la fesse droite. Tous lesquelles ponctuent les chairs ${ }^{28}$."

À en croire ce rapport, la fusillade fut certainement nourrie. Il est clair que ce braconnage constituait une véritable menace économique pour les fermiers des pêcheries qui étaient déjà tributaires des remontées de saumons. Les désastres causés par la furie des crues printanières et automnales suscitaient aussi des faillites.

Les faits de braconnage proliféraient dans les espaces qui prolongeaient l'intimité domestique. Les paysans de Guidel, de Rédéné accomplissaient tout simplement leur larcin près de leurs terres. Connaissant les moindres recoins de la campagne, ils pouvaient se dissimuler derrière

26. Arch. dép. des Côtes d'Armor, 108 J 42.

27. Arch. dép. du Finistère, 20 B 4437.

28. Idem, 20 B 4452. 
des talus ou cacher des filets trop encombrants. Les perquisitions démontrent que le domicile, souvent proche, voire sur la rive opposée, servait aussi à dissimuler les ustensiles de pêche. En 1728, une perquisition organisée au domicile de Joseph Le Clanche porta ses fruits puisqu'elle permit de découvrir deux filets et deux saumons ${ }^{29}$. À Quimper, Pierre Troussier, fermier des pêcheries de l'évêque débusque un filet caché dans les fourrés ${ }^{30}$. D'autres laissent dormir leurs filets impunément dans le cours de la rivière. En 1766, Joseph Le Gallo, arrache du Blavet un filet de 110 pieds de long chargé de saumons ${ }^{31}$. C'est parfois à coups de bâtons et armés de fusils que les " gorestiers " sont obligés de dissuader les braconniers qui viennent tendre leurs pièges ${ }^{32}$. Les braconniers disposent aussi d'une certaine forme de complicité locale. Il faut dire que les fermiers ne sont pas toujours très appréciés. En 1729, des témoins se liguent contre les fermiers de l'abbaye de Sainte-Croix au profit des braconniers. Ils entretiennent le mensonge ce qui entraîne l'incarcération des fermiers $^{33}$. On peut également supposer que ce silence ou ces faux témoignages étaient achetés et entretenus par de petits cadeaux occasionnels. Un saumon pouvait améliorer une table quotidienne. La violence de certains d'entre eux pouvait faire naître des craintes de vengeances.

\section{Sociologie des braconniers}

Le braconnier c'est d'abord un homme qui fait un usage illicite de son filet. Ensuite c'est souvent un homme violent, imprévisible surtout lorsqu'il est surpris en train d'accomplir son forfait. Ils sont souvent accusés d'avoir des mœurs légères et sont qualifiés de "coquins ", "d'effrontés " voir " d'effrontés déterminés ». Qu'ils soient appelés "fripons de saumons de nuit " ou encore "fieffés fripons de toutes choses ", ils inquiètent par leur dangerosité ${ }^{34}$. Ainsi, Jacques Teffany est qualifié de "déterminé et coquin dans l'âme " mais aussi " d'homme dangereux ${ }^{35}$ ". Yves Le Maou est réputé " dangereux et coquin ${ }^{36}$ ". Cette violence est d'abord verbale. Avant d'en venir aux mains, Le Clanche profère des insultes à l'encontre des fermiers des religieux de l'abbaye noire de Quimperlé. Ce n'est qu'après cet échange qu'il fond sur eux avec ses acolytes, armés de bâtons ferrés. Parfois même, ils font fuir les fermiers les plus déterminés ${ }^{37}$. Ces insultes ne viennent pas toujours des braconniers de la pire espèce. Parfois, ce sont les hommes d'un autre seigneur, réclamant les mêmes droits qui se chargent de cette basse besogne. À Quimperlé, ce sont les

29. Id.

30. Id., 1 G 97.

31. Arch. dép. du Morbihan, 60 H 67.

32. Arch. dép. du Finistère, 20 B 4441.

33. Idem, 20 B 4452.

34. Arch. dép. des Côtes d'Armor, 108 J 42.

35. Idem.

36. Id.

37. Arch. dép. du Finistère, 20 B 4452. 
hommes des moines blancs qui viennent harceler les pêcheurs de l'abbaye de Sainte-Croix sous leurs pêcheries ${ }^{38}$. Parfois, c'est le seigneur, poussé par la jalousie et la convoitise, qui se déplace en personne. En 1727, l'abbé de Saint-Maurice de Carnoët, accompagné de ses domestiques, vient vociférer sous les pêcheries. Il tente d'intimider les fermiers en brandissant sa croix pectorale tout en leur demandant "s'ils étoient capables pour disputer contre celui qui la portoit ${ }^{39}$ ». Intimider, injurier, terroriser, frapper et blesser telle est l'escalade de cette violence occasionnelle dont le saumon est le principal enjeu. Les braconniers ont intérêt à faire régner la terreur : elle éloigne les importuns et dissuade de porter plainte ou de témoigner. Le braconnage, puisqu'il est illicite, ne peut qu'être le fait des couches inférieures de la société. Pourtant, il apparaît au vu de quelques exemples que ces voleurs de saumons agissent parfois pour le compte de plus importants qu'eux.

En ce qui concerne les braconniers verbalisés après avoir volé des saumons sur les pêcheries du duc de Richelieu à Pontrieux, il apparaît qu'ils font tous partie de la juridiction et qu'ils habitent à proximité du lieu de leurs délits. Sur 24 braconniers recensés au milieu du XVIII ${ }^{\mathrm{e}}$ siècle, deux seulement sont marins, ce qui permet de supposer que le braconnage n'est pas uniquement l'apanage des pêcheurs; 12 sont artisans ou commerçants (Yves Floury, demeurant à Pontrieux est cordonnier, Jean Le Gras charpentier, Yves Le Maou boucher); 3 sont sergents gardes côtes et 2 sont laboureurs. Le plus surprenant est la quasi absence de cette frange flottante de la population en laquelle les autorités se plaisent à voir l'archétype du délinquant. Sur les 24 délinquants connus sur la place de Pontrieux, seul un d'entre eux est qualifié de vagabond : Pierre Morvan "surnommé marquis Morvan " est décrit comme un "vagabond, grant et bon corps trapu ${ }^{40}$ ". Cet exemple montre que cette délinquance caractérise l'ensemble de la société des petites villes bretonnes. Les braconniers qui contestent les droits de pêche et les pêcheries de l'abbaye de La Joie sont majoritairement des pêcheurs de Lochrist et de Carantec attirés par la forte concentration de poissons aux pieds des barrages. Les meuniers de l'autre rive n'hésitent pas à tendre des filets à l'entrée de leur bief. En 1766, Joseph Le Gallo, meunier et gorestier pour l'abbesse de La Joie se plaint de son voisin, Henri Thomas qui pratique cette pêche frauduleuse ${ }^{41}$. Par conséquent, il est assez difficile de brosser un tableau social précis de cette micro société des braconniers.

Braconner est un métier d'hommes. Dans les sources étudiées, les femmes n'apparaissent jamais, sauf pour le recel. Les braconniers sont le plus souvent de jeunes gens dans la force de l'âge qui dépassent très rarement la trentaine. Certains commencent tôt dans le métier comme Pierre Morvan de Pontrieux, arrêté à l'âge de 16 ou 17 ans ${ }^{42}$. Le plus souvent ils

38. Idem, 5 H 39.

39. Id.

40. Arch. dép. des Côtes d'Armor, 108 J 42.

41. Arch. dép. du Morbihan, 60 H 67.

42. Arch. dép. des Côtes d'Armor, 108 J 42. 
n'agissent pas seuls, à l'exception de quelques pêcheurs qui entrent en guerre contre l'autorité seigneuriale. En 1727, Guillaume Keraudren, pêcheur professionnel, défie sans cesse les droits et l'autorité de l'évêque de Cornouaille en tendant ses filets sous les pêcheries. C'est à grand renfort d'hommes armés de bâtons et de fusils qu'il faut le déloger et le contraindre à quitter les lieux ${ }^{43}$. La plupart du temps, les associés sont au nombre de deux ou trois personnes. À Pontrieux, dix composent des fratries et parfois les alliances gendre beau-frère font bon ménage. Vincent Le Gras et son frère Allain braconnent ensemble sur les rivages possédés par le duc de Richelieu ${ }^{44}$. Parfois, c'est le père qui initie ses fils. Les Le Clanche, malfaiteurs et braconniers connus à Quimperlé au XVIII ${ }^{\mathrm{e}}$ siècle commencent leur carrière de malfrats sous la houlette de leur père. Le 8 septembre 1728, les fermiers se plaignent des quatre hommes de cette famille qu'ils ont surpris à maintes reprises en train de relever leurs filets pour voler le poisson. Par mesure de représailles les braconniers coupent leurs filets. D'ailleurs la perquisition effectuée au domicile du père Joseph permet de les découvrir, lui et ses fils, en train de réparer deux filets ${ }^{45}$. Les actions en bandes organisées sont très rares. Elles restent le fait de petits bandits se cantonnant à des exactions très localisées. Le 14 avril 1729, les fermiers surprennent une bande de six braconniers en train de récolter les fruits de leurs nombreux coups de filets. Ces derniers sont formellement reconnus par les fermiers alors qu'il fait nuit noire ${ }^{46}$. C'est le signe que ces bandes sont rares et que les fermiers les connaissent. Toutefois, dissimulés dans les taillis, ils connaissent parfaitement les moindres recoins de la campagne quimperloise et la population les soutient, par crainte ou par intérêt : tous les témoins sont d'accord pour dire que Le Clanche avait perdu son taureau cette nuit-là. Ce qui, bien sûr, lui sert d'alibi. La frontière est mince entre ces manières musclées de pêcher en bande et le banditisme qui s'en prend aux personnes et aux biens. D'autant que ces derniers molestent les fermiers et pillent parfois leur matériel de pêche ou tout simplement se livrent à des actes de vandalisme en coupant leurs filets. Le chef de cette bande sera emprisonné à Quimper la même année, c'est bien la preuve que le cas de cette association de malfaiteurs inquiétait les autorités juridiques.

Ces braconniers travaillent en relation avec des poissonniers et des poissonnières soucieux d'acheter du saumon de contrebande au plus bas prix. Le 23 mai 1741, la dame Anne de La Roche, douairière de Kerstrat, en qualité de fermière de la pêcherie royale de Châteaulin, lance une procédure pour vols et pillages contre Jean et Jacques Salaun, père et fils, voituriers et marchands de poissons à Châteaulin ${ }^{47}$. Lors de l'information judiciaire les langues se délient et on apprend très vite comment

43. Arch. dép. du Finistère, 20 B 4441.

44. Arch. dép. des Côtes d'Armor, $108 \mathrm{~J} 42$.

45. Arch. dép. du Finistère, 20 B 4452.

46. Idem, 20 B 4437.

47. Id., B 2172. 
fonctionne la combine de nos deux compères. C'est en général le fils qui vient informer une des poissonnières attitrées de son père. La transaction se passe en dehors de la ville le plus souvent dans un champ. Une fois le poisson acheté, la receleuse le dissimule dans son panier. Ce manège ne tarde pas à rameuter d'autres poissonnières jalouses de leur consœur. Ce petit jeu commence à devenir dangereux et les Salaun décident de brouiller les cartes. Le lieu de rendez-vous est désormais fixé dans une maison sise sur la route de Châteaulin à Landerneau. Finalement, à force d'espionner, les autres poissonnières découvrent le subterfuge et ébruitent l'affaire. C'est bien la preuve qu'il existait des réseaux organisés et que l'usage des complicités locales était de rigueur. Toutefois, ce système ne pouvait que susciter les envies et les jalousies des personnes qui en étaient écartées. Ce marché parallèle faisait circuler de la main à la main une très grande quantité de saumons puisque les témoignages parlent d'une bonne quarantaine de poissons.

Acte de banditisme caractérisé, le braconnage touchait toutes les couches de la société de l'Ancien Régime. Les seigneurs obligeaient leurs domestiques et autres valets à accomplir les basses besognes. Cette fraude est avant tout une déviance de proximité. En général, les braconniers tirent leurs connaissances du fait qu'ils demeurent dans la paroisse où ils accomplissent leurs larcins ou dans des villages limitrophes. Craints, car souvent violents, ils jouissent du silence et de la complicité des autres villageois. Ils constituent pour certains une sorte de cancer qui vient ronger les derniers édifices des privilèges seigneuriaux, jugés injustes. C'est sur les hommes des seigneurs que se cristallisent toutes les rancunes et toutes les haines. Souvent associés, les braconniers opèrent à plusieurs, de jour comme de nuit et alimentent un marché parallèle qu'ils contrôlent. D'autres, agissant pour leur propre compte, s'assurent ainsi un complément alimentaire de choix. 


\section{RESUME}

Sous l'Ancien Régime, la pêche du saumon, trés réglementée, était un privilège seigneurial. Certaines sources confirment son abondance et cela dès le $\mathrm{xvI}^{\mathrm{e}}$ siècle. Poisson royal par excellence, sa chair était trés estimée et son prix en faisait un met luxueux. Sur les marchés, ses principaux concurrents étaient le brochet et surtout le turbot. Sa pêche était louée et le commerce de sa chair procurait de jolis profits.

Mais le caractère exceptionnel de ce poisson en faisait une espèce convoitée. Tous ces facteurs expliquent le caractère endémique du braconnage. Les braconniers opéraient de jour comme de nuit, en bande ou seul, en toute impunité. Délit de proximité par excellence, le braconnage n'était pas que l'apanage des plus pauvres. Certains seigneurs, par pure jalousie, n'hésitaient pas à mandater leurs valets pour accomplir leurs méfaits. Parfois redoutés, car violents et dangereux, les braconniers jouissaient de la complicité d'un voisinage craintif.

\section{ABSTRACT}

Under Ancien Regime, the fishing of salmon, very regulated, was a nobiliary privilege. Certain files confirm its abundance and that as of XVIt century. Noble fish pre-eminently, his flesh was very estimated and its price made luxurious dish of it. On the markets, its principal competitors were the pike and especially the turbot. Its fishing was rented and the trade of its flesh got pretty profits.

But the salmon was also a coveted fish. It was the subject of an endemic poaching. The poachers were not only the poor. Certain lords, by pure jealousy, did not hesitate to elect their employees to achieve their misdeeds. Sometimes dreaded bus violent one an dangerous, they enjoyed the complicity of an apprehensive vicinity. 
